Notes from ISC Data Users

\title{
Using ISC Data
}

\section{Jens Havskov and Kathrin Lieser}

University of Bergen, Department of Earth Science, Bergen, Norway

International Seismological Centre, Thatcham, UK

Excerpt from the

Summary of the Bulletin of the International Seismological Centre:

Havskov, J. and Lieser, K. (2021), Using ISC Data, Summ. Bull. Internatl. Seismol. Cent., January June 2019, 56(I), pp. 30-46, https://doi.org/10.31905/L2IR6ZNA. 


\section{5 \\ Notes from ISC Data Users}

\subsection{Using ISC Data}

Jens Havskov ${ }^{1}$ and Kathrin Lieser ${ }^{2}$

(1) University of Bergen, Department of Earth Science, Bergen, Norway

(2) International Seismological Centre, Thatcham, UK

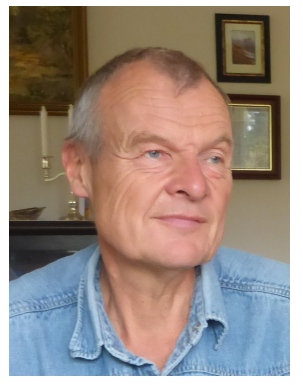

Jens Havskov

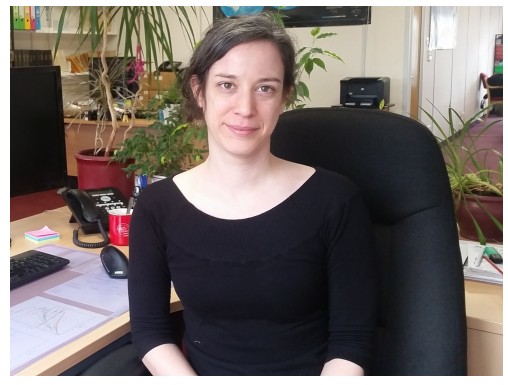

Kathrin Lieser

It is well known the ISC has the most complete database of seismic event parameter data available anywhere and it also has the most complete catalogue of epicenters. The ISC will receive and store any data that is submitted so the ISC can also function as a useful backup for individual agencies. All that data is available online. Currently there are more than 27000 stations registered in the International Seismograph Station Registry and there has been a large increase in data received by ISC since it was founded in 1964 (see Figs. 7.3 and 7.7). To review all data available today would be too timeconsuming and therefore, currently only events larger than magnitude 3.5 and a few others smaller than 3.5 under specific conditions (see Chapter 10.1 ISC Operational Procedures in Appendix, p. 110) are reviewed and/or relocated and magnitudes recalculated. For unreviewed events, only the hypocentres and magnitudes from the submitting agencies are given while for reviewed events ISC magnitudes and hypocentres are given in addition, if available. This of course is mostly the case for distant events. In addition, not all data is used in the reprocessing such as some amplitudes and back azimuths. While the hypocentres and magnitudes calculated by the ISC are very uniform, some associated data is less so (e.g., amplitudes that are reported by different agencies with different standards). Therefore, extracting a data set comprised of reviewed and unreviewed events will result in a non uniform data set and reprocessing it to make it uniform requires some filtering. In this note we will give some guidelines on how this can be done and give some examples.

The user can take out data from the ISC Bulletin in two formats: ISF (see Example 1) (http://www. isc . ac.uk/standards/isf/) and QuakeMl (https://quake.ethz.ch/quakeml/). The extracted ISC data will be illustrated using the most used ISF format. Some of the converted data is tested with SEISAN (Havskov et al., 2020) which has software to convert ISF format to SEISAN (http://seisan.info). 


\subsubsection{Hypocentres}

How the ISC is processing: The ISC reviews all events above M3.5 and certain events between 2.5 and 3.5 dependent on azimuthal gap, number of reported phases and number of reporting agencies. However, during review, tiny local events can also become reviewed events, e.g., when it is needed to move phases out into larger events. But not all reviewed events have an ISC hypocentre. At least two different agencies must report phases and an azimuthal gap of less than 315 degrees is required for an event to qualify for an ISC solution. If the event does not meet the criteria for an ISC solution the event is fixed to an agency hypocentre, i.e. residuals are calculated with the ak135 velocity model (Kennett et al., 1995) where the hypocentre parameter of an agency are used (origin time, RMS, latitude, longitude, depth etc.) and the phase names are changed according to ak135. If several hypocentres are available one will be designated the prime hypocentre. If an ISC solution is available this will usually be the prime hypocentre (see Example 1), except for some rare cases where the IASPEI solution for reference events was set as prime for nuclear explosions. If there is no ISC solution the prime is found by a score based on the network coverage. Should this not be available it is essentially random.

There are some exceptions to these rules regarding IDC (International Data Center of CTBTO). All events reported by IDC are reviewed and unless IDC is the only hypocentre author with less than six associated seismic phases in an event, the ISC will try to calculate an ISC hypocentre.

In short: every ISC solution is reviewed but not all reviewed events have an ISC solution. Reviewed events will show residuals according to ak135 while unreviewed events are not relocated and will not show residuals even if the reporting agency has reported any residuals. For more details see ISC Operational Procedures (Appendix).

If, for a particular area, the user takes out a mixture of reviewed and unreviewed events, the data set will not be uniform since different programs and earth models might have been used by the several reporting agencies. To create a uniform data set it must be relocated as not all reported hypocentres have been calculated with ak135 and unreviewed events do not show any residuals. That can lead to problems with phase names, see next section. Note that when searching the ISC Bulletin for a particular area and an event has several hypocentres, the event will be selected if any of the hypocentres are within the selected area. Similarly, if any of the magnitudes are within the given range, the event will be selected. However, there is an option to only take magnitudes determined by a specific author into account.

Recommendation: Use the prime solution. However be aware that the prime hypocentre in an unreviewed event with several reporting agencies might not always be the best fitting solution and the quality can only be judged by reviewing it after relocation. It should also be mentioned that not all agencies send all of their station data to the ISC and an agency's hypocentre might have been calculated with more stations available to the local agency than is shown in the ISC Bulletin.

\subsubsection{Seismic Phases}

The IASPEI Commission on Seismological Observation and Interpretation (Storchak et al., 2003, http: //www.isc.ac.uk/standards/phases/) has given a recommended list of official phases that should be used in reporting. The identification of phases varies a great deal between the currently 150 reporting 
agencies. The ISC location program, ISCloc (Bondar and Storchak, 2011) will reinterpret the phase type so it best fits the ak135 model used for location which will make the phase identification more uniform for reviewed events. The original phase names provided by the agencies are stored and the user can get them by extracting data in QuakeMl format but not in the ISF format. The original data files submitted to the ISC can be found online on the agency web pages of the ISC web site: http://www.isc.ac.uk/iscbulletin/agencies/.

The ISC uses a relative weighting scheme to ensure that arrivals picked less reliably or prone to phase identification errors are down-weighted in the location algorithm (see Bondar and Storchak, 2011; ISCloc manual (http://www.isc.ac.uk/iscbulletin/iscloc/) and ISC Operational Procedures (Appendix)). Reported weights and weights calculated by ISCloc are not provided and the only indication on a high or low weight is if the phase is flagged as time defining or not. Note that phase weights reported by the agencies are not used.

ISCloc is allowed to re-interpret phases in every distance range, e.g., $\mathrm{P}$ phases can become $\mathrm{Pn}, \mathrm{Pb}, \mathrm{Pg}$, PP, PnPn, PKiKP, Pdif, PcP, PKP(ab/bc/df) etc as well as depth phases, with a similar set up for $\mathrm{S}$ phases. There are certain rules to that, e.g., depth phases cannot be set as first arrivals by ISCloc or $\mathrm{P}$ phases cannot be renamed as S phases. An analyst can fix a phase to every available phase name manually though. Some agencies report their phases as just P, even if they are actually a PP, PKPbc or PKiKP phase and thus ISCloc needs to be flexible. Obviously, this can go wrong and will be fixed during review by an analyst. Only P and S type phases are used for location and phases like Lg and T are not used. If residuals are larger than $60 \mathrm{~s}$ the phase is treated as unidentified. For more details see ISCloc manual (http://www.isc.ac.uk/iscbulletin/iscloc/). ISCloc will take all IASPEI phases into account but not all phases necessarily become time defining. This depends on the weighting algorithm in ISCloc, e.g., very large residuals are rejected. Phases not contributing (meaning weighted out) will not have a time defining flag but the residuals will be calculated. Example 1 shows an event where some phases are time-defining while others are not: Pn on station SRK with a residual of $-5.1 \mathrm{~s}$ was not used in the location, while PETK Pn with -1.5 s residual contributed to the solution as can be seen by the time defining flag $\mathrm{T}$ in column Def.

Example 1: Event where some phases are time-defining while others are not (ISF format). The abbreviations and units in this and the following (ISF) examples are: Err - origin time error, Smaj and Smin - semi major and minor axis of the epicenter error ellipse in $\mathrm{km}, \mathrm{Az}$ - azimuth of error ellipse (degree), Depth $(\mathrm{km})$, f for fixed, Ndef - number of defining phases, Nsta - number of defining stations, Gap - azimuthal gap (degree), Mdist - distance to closest station (degree), Qual (hypocentre) - analysis type and location method, Author - author of origin, Sta - station, Dist - epicentral distance (degree), EvAz - azimuth from event to station (degree), Tres - travel time residual (s), Azim - back azimuth (degree), Slow - slowness (s/degree), Sres - slowness residual (s/degree), Def - T is time defining flag, SNR - signal to noise ratio, Amp - amplitude (nm), Per period of amplitude (s), Qual (phases) - direction of motion, manual (m) or automatic (a), onset quality, Magnitude - showing both the type and the value. Standards and formats can be found here: http://www.isc.ac.uk/standards/ 

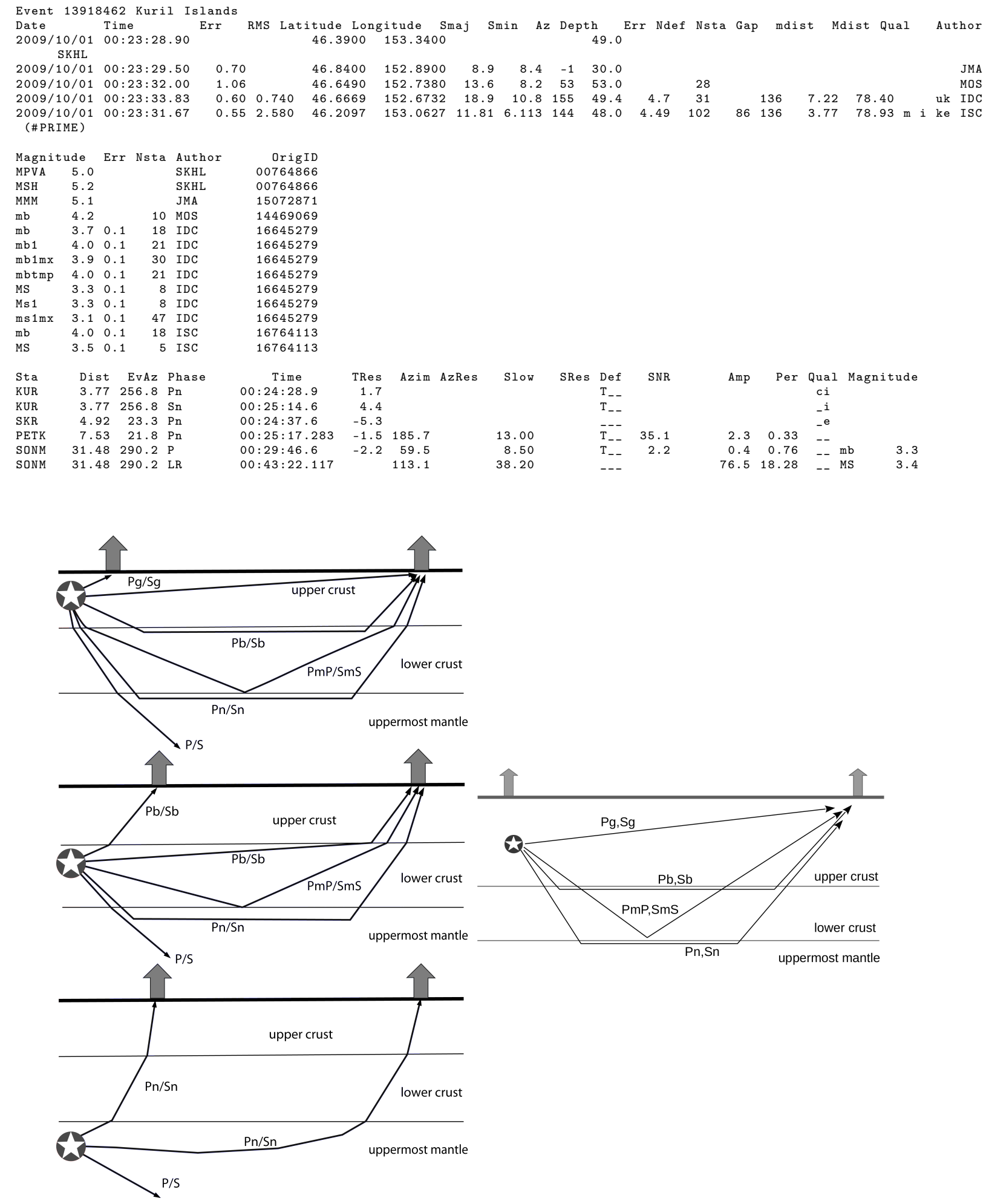

Figure 5.1: Left: Phase definition in the ak135 model (after Storchak et al., 2003, 2011; Schweitzer et al., 2019), right: traditional phase definitions (e.g. Aki and Richards, 2002; Stein and Wysession, 2003).

For some local phases the change of phase names gives an undesirable side effect. At short distances the main phases are $\mathrm{Pg}, \mathrm{Pb}$ and $\mathrm{Pn}$ which traditionally are identified as seen in Figure 5.1 with $\mathrm{Pn}$ as a refracted wave along the Moho. However, the IASPEI definition of Pn follows the ak135 notation that includes any $\mathrm{P}$ wave bottoming in the uppermost mantle or an up-going $\mathrm{P}$ wave from a source in the uppermost mantle (http://www.isc.ac.uk/standards/phases/). This implies that for some 
distance/depths both $\mathrm{Pb}$ and $\mathrm{Pn}$, in the ak135 definition, are what most location program would call $\mathrm{Pg}$ or $\mathrm{P}$. The $\mathrm{Pg}$ phase in ak135 is what is traditionally called Pg. This change of definition from earlier practice was decided by the IASPEI Working Group on Standard Phase Names because the corresponding ak135 code did not differentiate between the classic Pn travelling as a headwave alongside the Moho and the branch of direct $\mathrm{P}$ that leaves a source in the uppermost mantle (Dmitry Storchak, personal communication).

For local earthquakes, the user will mostly use a standard location program (such as Hypoinverse (Klein, 2002) or Hypocenter used in SEISAN (Lienert and Havskov, 1995)) using a flat layer velocity model. Having the phases identified as $\mathrm{Pb}$ or $\mathrm{Pn}$ will then imply an identification which might not be in accordance with the local model or which cannot be calculated (Pn if the source is below Moho, $\mathrm{Pb}$ if the source is below Conrad). The $\mathrm{Pb}$ phase is rarely observed in practice and the corresponding Conrad layer might not be present in all models. So for local use $\mathrm{Pb}$ and $\mathrm{Pn}$ should be relabelled as $\mathrm{P}$ in order to get reliable locations (see Examples 2-4). The same is the case for the corresponding S-phases.

Example 2: The agency identified phases are shown in parenthesis. The agency phase identification is what traditionally would have been expected and the nearest station show Pg and Sg. In this case changing all $\mathrm{Pn} /$ Sn-phases to $\mathrm{P}$ and $\mathrm{S}$ will, when relocating, again identify the first two phases as g-types.

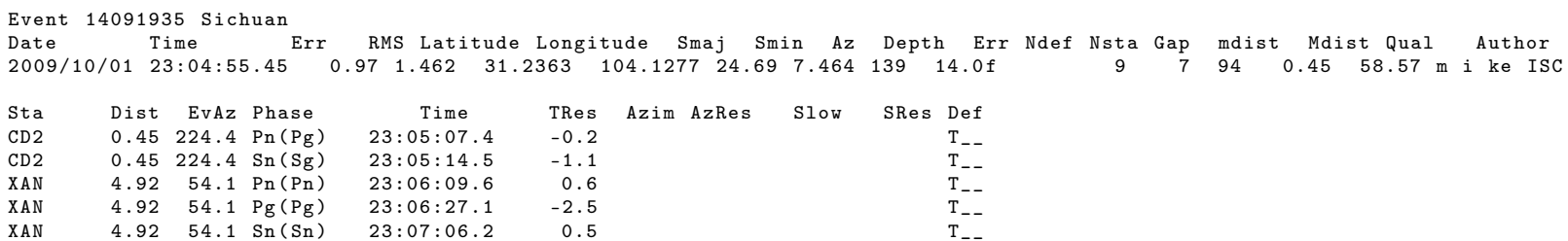

Example 3: In this event, all phases are labelled as $\mathrm{Pn} / \mathrm{Sn}$ and a standard location program will put the event above Moho unless the program ignores the $\mathrm{n}$. The original phases reported were all $\mathrm{P}$ and S. By removing the $\mathrm{n}$, Hypocenter locates the event at $160 \mathrm{~km}$ depth. We do not know the local model or program used to get the agency's $86 \mathrm{~km}$ depth. Removing station JHO gives a $40 \mathrm{~km}$ depth so the depth is not well defined. These phases would traditionally have been called $\mathrm{P} / \mathrm{S}$. Note that the time defining flag is not set since the ISC did not locate the event and only calculated the residuals with the location fixed to the JMA solution.

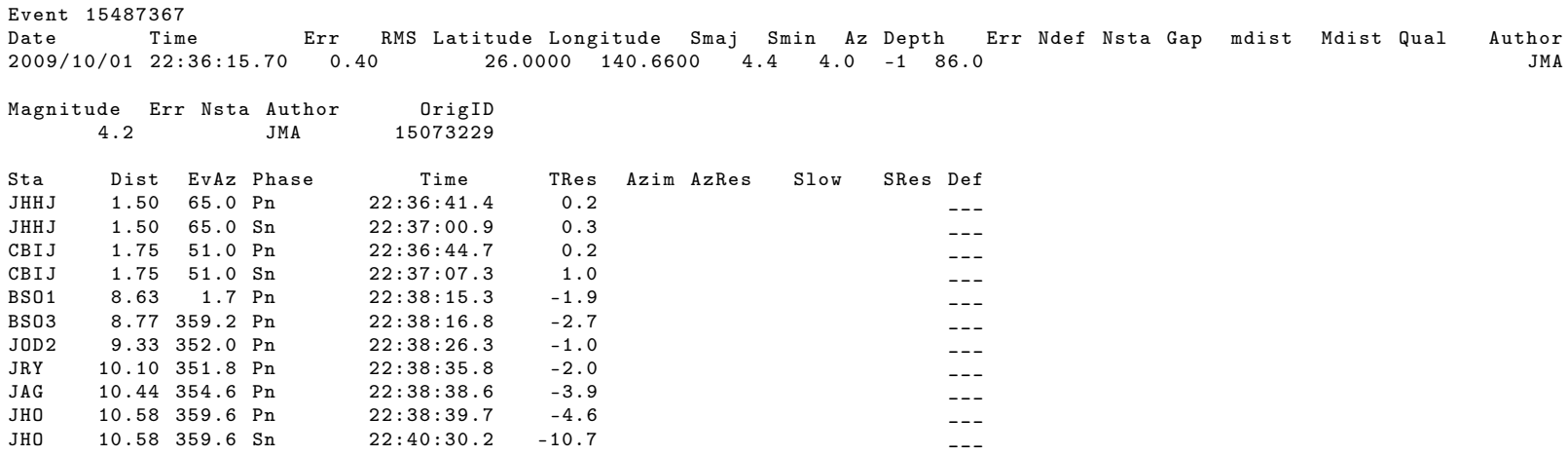

Example 4: The nearest station in this example reports $\mathrm{Pb}$ (VLS) and a station further away reports Pg (AMT). Trying to relocate this event with Hypocenter and the ak135 model will ignore the first 
station (if flag for enforcing $\mathrm{Pb}$ and $\mathrm{Pn}$ is set) since it is too close for $\mathrm{Pb}$. When using only $\mathrm{P}$ and $\mathrm{S}$, all stations will be used. For more distant stations, $\mathrm{Pn}$ will be the first arrival instead of $\mathrm{Pb}$, the phase at the closest station will be Pg and the RMS will be smaller compared to forcing the arrivals to be $\mathrm{Pb}$. This shows that it can be problematic to label first arrivals $\mathrm{Pg}, \mathrm{Pb}$ and $\mathrm{Pn}$.

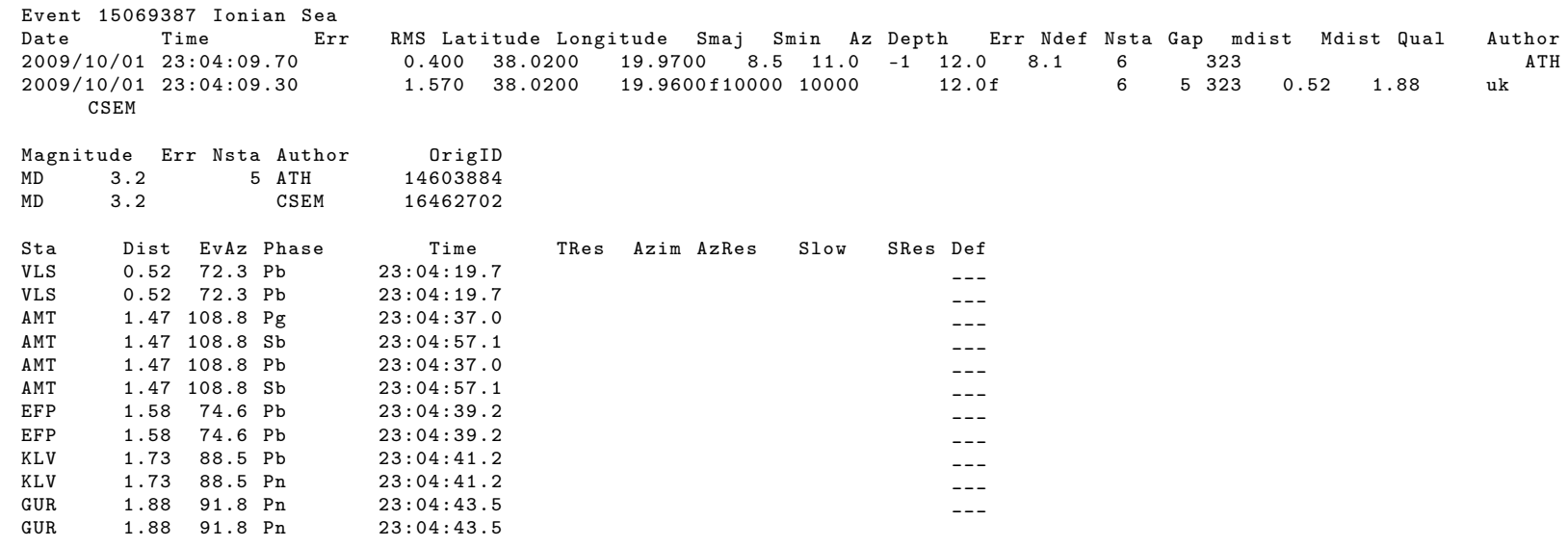

Below is the SEISAN relocation forcing $\mathrm{Pb}, \mathrm{Sb}$ and $\mathrm{Pn}$. PN2 means refracted from interface 2 (Conrad) and PN3 is refracted from layer 3 (Moho).

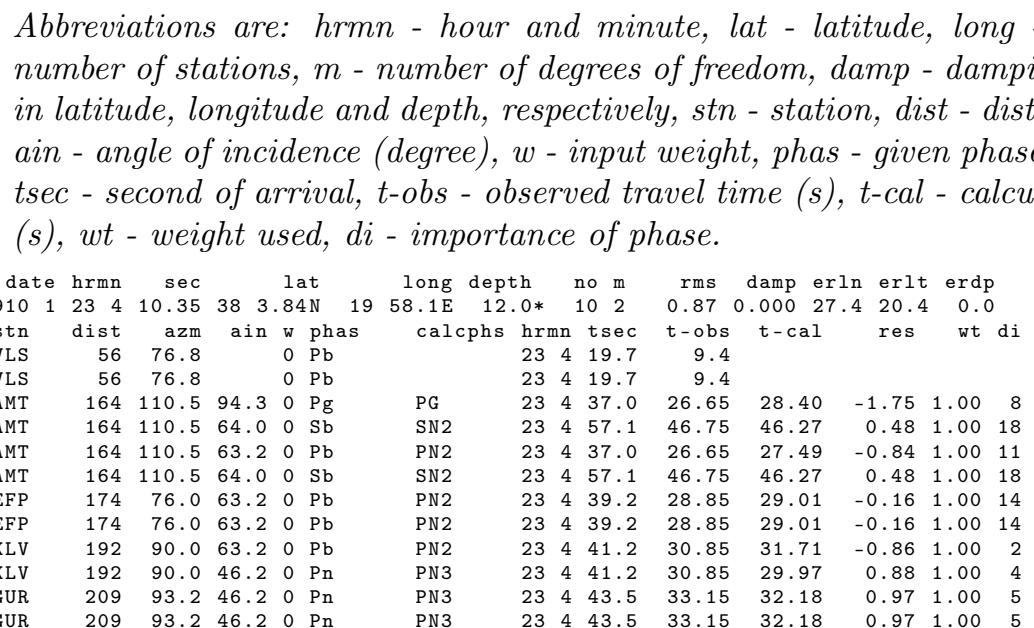

SEISAN output using just $\mathrm{P}$ and $\mathrm{S}$ :

\begin{tabular}{|c|c|c|c|c|c|c|c|c|c|c|c|c|c|c|}
\hline date & hrmn & sec & & $1 \mathrm{a}$ & & long & & no $\mathrm{m}$ & $\mathrm{rms}$ & damp & erln & erlt & $\operatorname{erd}_{\mathrm{F}}$ & \\
\hline 9101 & 234 & 11.59 & 38 & 6.32 & 20 & $1.6 \mathrm{E}$ & & & 0.55 & 0.000 & 16.1 & 10.2 & 0.0 & \\
\hline stn & dist & azm & ain & a $w$ & phas & calcphs & hrmn & $n$ tsec & $t$-obs & $s \quad t-c a$ & & res & wt & $\mathrm{di}$ \\
\hline VLS & 50 & 80.7 & 103.9 & 0 & $\mathrm{P}$ & PG & 234 & 19.7 & 8.11 & 8.8 & & -0.77 & 1.00 & 12 \\
\hline VLS & 50 & 80.7 & 103.9 & 0 & $\mathrm{P}$ & & 23 & 19.7 & 8.11 & 8.8 & & -0.77 & 1.00 & 12 \\
\hline AMT & 161 & 112.7 & 46.2 & 20 & $\mathrm{P}$ & PN3 & 23 & 37.0 & 25.41 & 26.1 & & -0.75 & 1.00 & 11 \\
\hline AMT & 161 & 112.7 & 50.6 & 50 & $\mathrm{~s}$ & SN3 & 23 & 57.1 & 45.51 & 45.1 & & 0.32 & 1.00 & 19 \\
\hline & & 12.7 & 46.2 & 20 & $\mathrm{P}$ & & 234 & & & & & -0.75 & 1.00 & 11 \\
\hline IMT & 161 & 112.7 & 50.6 & 50 & $\mathrm{~s}$ & SN3 & 234 & 57.1 & 45.51 & 45.1 & & 0.32 & 1.00 & 19 \\
\hline EPP & 168 & 77.2 & 46.2 & 20 & $\mathrm{P}$ & PN3 & 23 & 39.2 & 27.61 & 27.0 & & 0.61 & 1.00 & \\
\hline$F P$ & & 77.2 & 46.2 & 20 & P & & 234 & 39.2 & 61 & 27.0 & & 0.61 & 1.00 & \\
\hline KLV & 186 & 91.5 & 46.2 & 20 & $\mathrm{P}$ & PN3 & 234 & 41.2 & 29.61 & 29.3 & & 0.27 & 1.00 & \\
\hline $\mathrm{LV}$ & & 91.5 & 46.2 & 20 & $\mathrm{P}$ & & 23 & 41.2 & & 29 & & 0.27 & 1.00 & \\
\hline GUR & 204 & 94.6 & 46.2 & 0 & $\mathrm{P}$ & PN3 & 23 & 43.5 & .91 & 31.5 & & 0.33 & 1.00 & \\
\hline GUR & 204 & 94.6 & 46.2 & 20 & & PN3 & 234 & 43.5 & 31.91 & 31.5 & & 0.33 & 1.00 & \\
\hline
\end{tabular}

To use the most reliable data it is recommended to give a full weight to phases that are time defining in an ISC solution as these phases will have been identified by ISCloc and will show acceptable residuals. However, non-defining phases should not be dismissed because of their larger residuals or no residuals (meaning that ISCloc was not able to identify this phase) as they might be a good fit when using 
another velocity model. ISCloc imposes hard limits on distance and depth, e.g., Pg phases above around 10 degrees distance will not fit ak135 and show either no or large residuals. Another example for this is $\mathrm{PKPbc}$ which can be misidentified outside its defined distance range as a different phase by ISCloc and show large residuals. ISC analysts then fix the phase manually back to PKPbc, which will result in no residual being shown as it cannot be calculated. Also note that depth phases will only be time defining when they are accompanied by a $\mathrm{P}$ phase on the same station. Thus, depth phases can be a perfectly good pick whilst being non-defining. In some cases, phases can have good residuals but do not contribute to an ISC solution yet as ISCloc stops after a certain number of iterations. These phases will likely become time defining after another run of ISCloc, which is usually done by ISC analysts during review if necessary. Nevertheless, some phases in the ISC Bulletin might have good residuals while being non-defining due to this effect.

For events that are not reviewed by the ISC there are no residuals in the ISC Bulletin. For these events all phases have to be used for relocation since we have no idea which phases were used by the local agency. However, as there is often not a consistent practice when picking $\mathrm{Pg}, \mathrm{Pb}$ and $\mathrm{Pn}$ and corresponding $\mathrm{S}$ it might be necessary to also relabel these phases to just $\mathrm{P}$ and $\mathrm{S}$.

Recommendation: For events relocated by the ISC, use all phases with a time defining flag. Other phases, from the same event, without a time defining flag but with a residual, should be included with a low weight. Phases without residuals should be included with zero weight so they can be evaluated after relocation. ISC reviewed events which are fixed to the agency location and reported with ak135 residuals should all be used since there is no time defining flag. For events not reviewed and located by the ISC, use all phases. Consider relabelling all crustal phases to P and S.

\subsubsection{Back Azimuth and Slowness}

The back azimuth (BAZ) can be useful for improving the epicenter location, particular for small events. The ISC reports the back azimuth determined by an agency but ISCloc does not use it in calculating locations and thus there is no quality check. Back azimuth from arrays are usually OK, at least for $\mathrm{P}$, and could potentially be used. Occasionally, ISC analysts make use of back azimuth and slowness from arrays to confirm the phase association to an event if the phase readings are reported without a hypocentre. Slowness can be converted to apparent velocity and is useful for phase identification. However, currently the back azimuth must be removed or weighted out to not corrupt the relocation. Slowness is not used by many programs and, even for arrays, can be wrong.

Example 5: BAZ from arrays fit within about $+/-20$ degrees. For H11S2, the back azimuth fits well but the slowness is completely wrong as a T-wave takes more than $0.7 \mathrm{~s}$ to travel one degree. So be aware that automatic data from arrays might not always be reliable. Station AFI gives 3 different values for back azimuth where only 227 is correct.

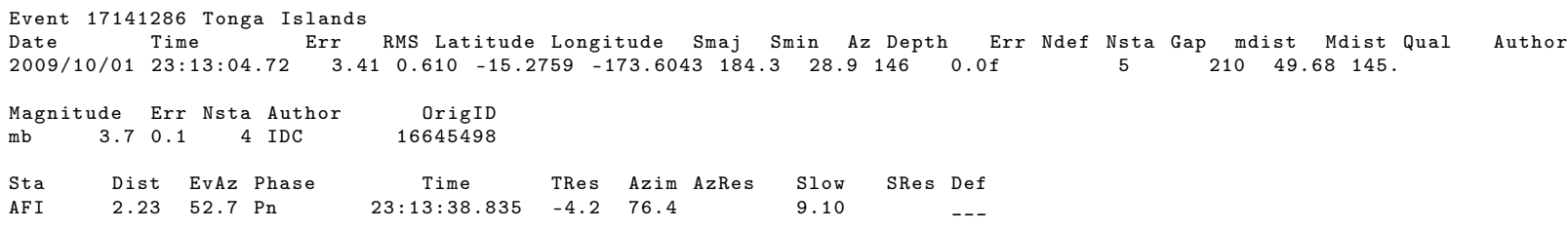




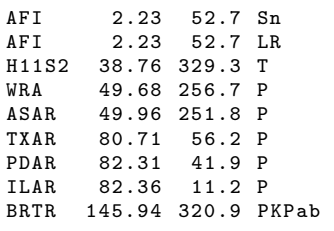

$\begin{array}{lrr}23: 13: 58.435 & -12.9 & 227.1 \\ 23: 14: 04.0 & & 183.6 \\ 00: 01: 16.715 & & 152.1 \\ 23: 21: 58.55 & -0.3 & 89.5 \\ 23: 22: 00.7 & -0.3 & 87.7 \\ 23: 25: 23.15 & 2.9 & 224.0 \\ 23: 25: 28.65 & 0.0 & 208.1 \\ 23: 25: 27.5 & -0.6 & 209.5 \\ 23: 32: 47.1 & -0.2 & 118.6\end{array}$

$\begin{aligned} 22.50 & --- \\ 26.60 & --- \\ 0.70 & --- \\ 6.90 & --- \\ 7.80 & --- \\ 5.20 & --- \\ 5.00 & --- \\ 7.10 & --- \\ 1.50 & ---\end{aligned}$

Example 6: This event was not relocated by the ISC. Relocating it with SEISAN without using BAZ, gives nearly the same solution as IDC. Including the back azimuth from the most reliable P phases changes the location slightly but also shows that the BAZ values are not very reliable with up to 40 degree residuals on $\mathrm{P}$ and up to 98 degrees on other phases.

Abbreviations: IDC - IDC location, SEISAN - SEISAN location without BAZ, - -BAZ - SEISAN location with BAZ.

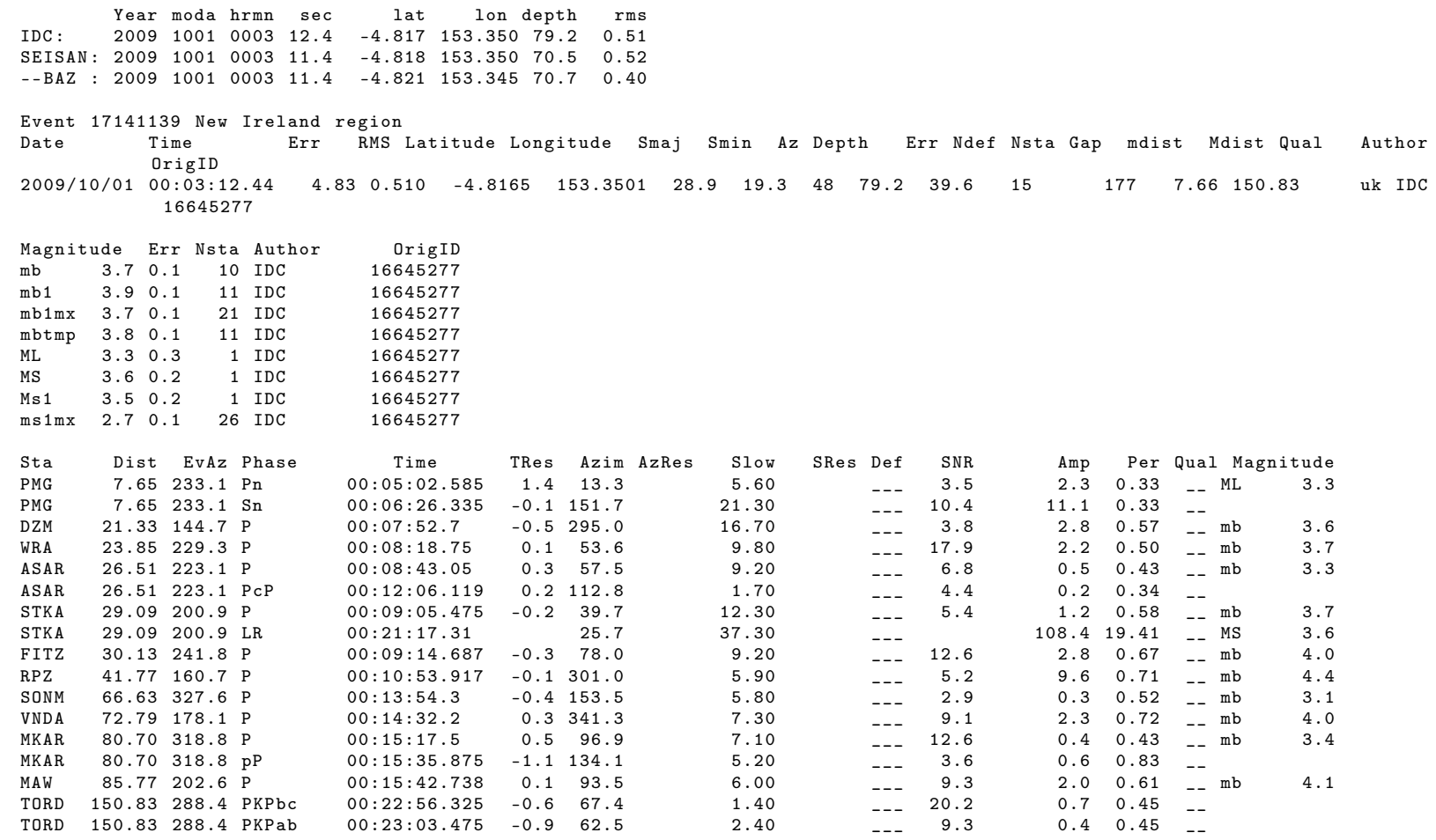

SEISAN BAZ residuals. T-obs, $t$-cal and res are now in degrees.

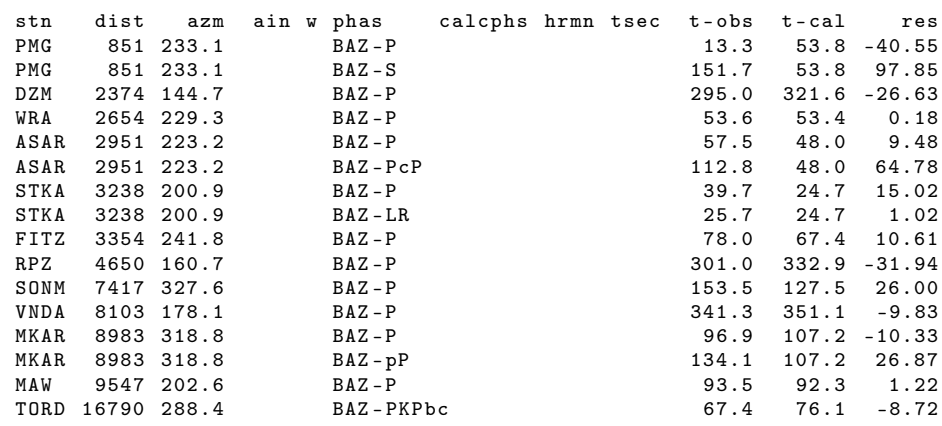

Recommendation: Weight out back azimuth or calculate residual and include if they fit. Often they do not. 


\subsubsection{Amplitudes}

Amplitudes can cause a lot of confusion since different standards have been used and it is not always clear what correction has been applied by different agencies to produce the amplitude readings. This becomes very complex for magnitudes not calculated by the ISC. Amplitudes can be reported together with the phase reading and in that case there is no information on the time of the observations. Or they can be reported as 'amplitude phases'. Amplitude readings that follow the standard of the IASPEI Working Group on Magnitudes seem to be mostly correct in the ISC Bulletin (IASPEI; Bormann and Dewey, 2014). The unit is supposed to be nm ground displacement if the names include an A (such as AML) or $\mathrm{nm} / \mathrm{s}$ if the name has a $\mathrm{V}$ (such as IVmBB), see http://www.isc.ac.uk/standards/phases/ \#amplitude. In addition, the ISC accepts amplitudes with the phase names P, pP, sP, AMB and pmax. Ideally only the latest IASPEI recommendation names should be used (it has been the standard for 8 years now) but that would severely limit the available data. The ISC only use amplitudes for mb and MS calculations so these amplitudes are checked and amplitudes that appear to be faulty are not used in the average. This is done by examining the station magnitude distribution (for mb and MS) during review for each event and spotting outliers and patterns. Usually the alpha trimmed median applied by ISCloc will dismiss outliers during magnitude calculations. Currently 39 types of magnitudes are reported to the ISC (see Section 7.5, p.66) and since the ISC is only calculating mb and MS, the input data (mainly amplitudes) for the remaining magnitudes are not checked.

\section{$\mathrm{Ml}$}

$\mathrm{Ml}$ is not recalculated by the ISC and there seem to be many non standard amplitudes reported. In addition $\mathrm{Ml}$ scales for different regions vary so there is no way of calculating correct $\mathrm{Ml}$ without using different scales from different regions. The IASPEI standard (Bormann and Dewey, 2014) requires to calculate maximum ground displacement in nanometres using a filter that simulates the Wood Anderson (WA) instrument response. The magnitude relation is:

$$
M l_{\text {iasp }}=\log (A)+1.11 \log (R)+0.00189 R-2.09,
$$

where $A$ is ground displacement in $\mathrm{nm}$ and $\mathrm{R}$ hypocentral distance in $\mathrm{km}$.

So in order to use amplitudes for Ml calculations some selection must be done. If the standard IASPEI notation IAML is used the amplitudes seem OK in most cases. AML is used for non-standard displacement amplitude measurements and some of these do not fit well. Many amplitudes reported with the $\mathrm{S}$ or Sg phases also seem OK, but there are examples where they are off by a factor of e.g., 1000 so there is no guarantee they are correct. In a few cases, the amplitudes have been reported in mm on a Wood Anderson seismogram instead of nm ground displacement (ground displacement $=$ WA displacement/2080). The ISC will convert the reported amplitudes to $\mathrm{nm}$ when parsing data into the database as this is the ISF standard unit for amplitudes. However, the ISC does not convert from WA to ground displacement as this cannot be reliably done with the various non-standard reported amplitudes. So be aware that amplitudes for $\mathrm{Ml}$ are given in $\mathrm{nm}$ but do not necessarily reflect ground displacement but could be the amplitude in $\mathrm{nm}$ on a Wood Anderson seismogram. Unfortunately, data are sometimes reported in the wrong unit. In obvious cases, such as values consistently being off by 1000 , this will be fixed if spotted by the ISC. Maximum amplitude is often reported on both P and S for short distances 
but there is no standard magnitude scale which can be used with these P-amplitudes. Amplitudes for $\mathrm{Ml}$ are not checked by the ISC, however it seems that amplitudes from trustworthy agencies are OK. IMS (International Monitoring System by CTBTO) arrays may report amplitudes that they use for Ml. However often they cannot be used since they may be non standard, often reported on both $\mathrm{P}$ and $\mathrm{S}$ beams so they give a wrong $\mathrm{Ml}$ if used with the standard scale. Amplitudes reported with AML seem mostly correct, but not always, see Examples 7-11. So in the end, only a fraction of the amplitudes for $\mathrm{Ml}$ reported by the ISC can generally be relied upon.

Example 7: The amplitudes seem to be too large by a factor of 1000 when initially calculating $\mathrm{Ml}$ and might have been reported in nm and not in micrometre as was indicated, but they were likely reported as Wood Anderson amplitudes. The comment says that amplitudes are in micrometres. However, when parsing the data into the ISC database amplitudes are converted into nanometres and comments like these should be ignored. The ISC aims to remove those comments and for the example below (and other events) this has now been done.

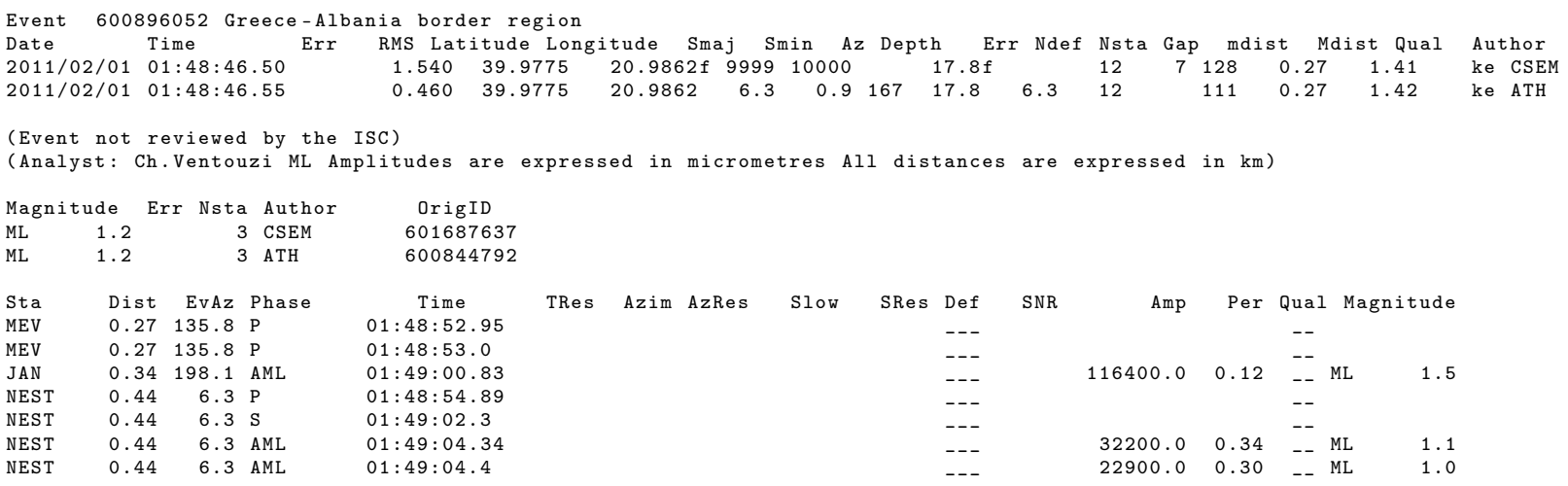

Table 5.1: Calculating $M l$ assuming amplitudes reported as ground displacement (Ml_iasp) gives the wrong magnitudes while assuming Wood Anderson seismogram amplitudes by dividing the amplitude by 2080 (ML_iasp_WA) yields the reported magnitudes. Amp is amplitude and $R$ is hypocentral distance.

\begin{tabular}{ccccccc} 
Station & Phase & $\mathbf{R}(\mathbf{k m})$ & Amp $\mathbf{( n m})$ & Reported Ml & Ml_iasp & Ml_iasp_WA \\
\hline \hline JAN & AML & 42 & 116,400 & 1.5 & 4.9 & 1.6 \\
NEST & AML & 52 & 32,200 & 1.1 & 4.4 & 1.1 \\
NEST & AML & 52 & 22,900 & 1.0 & 4.3 & 1.0 \\
\hline
\end{tabular}

Example 8: Both AML and IAML amplitudes are reported for the same station by two different agencies and only IAML reported in ground displacement. Although both station magnitudes are a bit high compared to the reported network magnitudes.

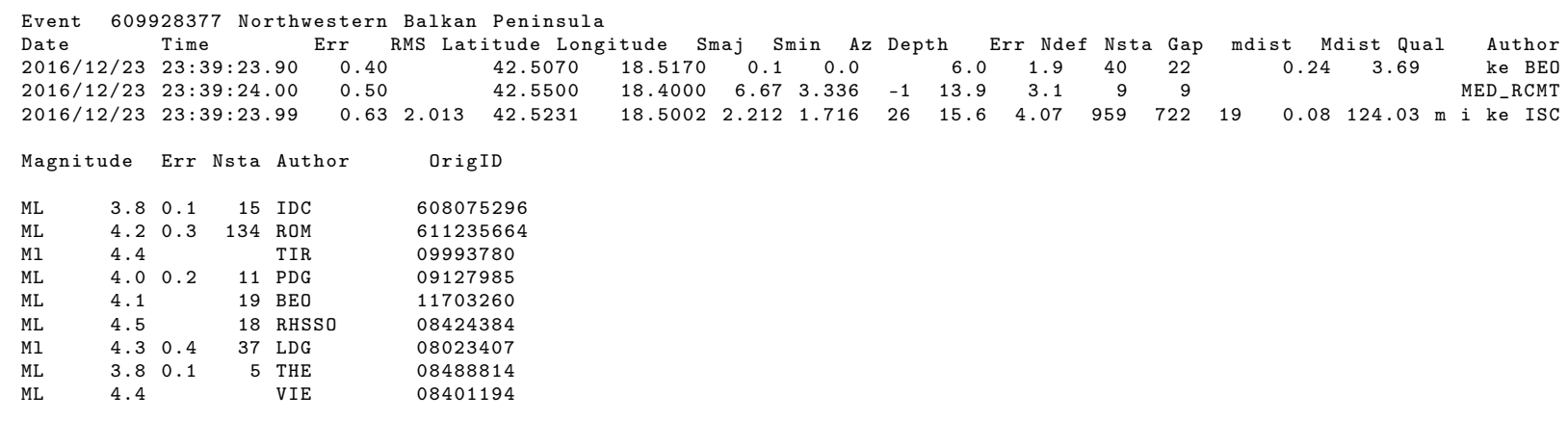




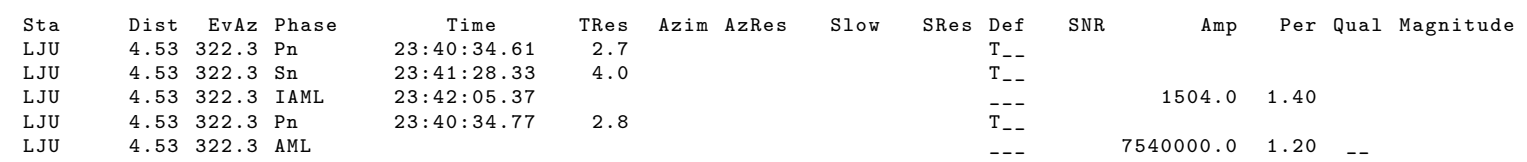

Table 5.2: Calculating $M l$ assuming amplitudes reported as ground displacement (Ml_iasp) gives the wrong magnitude for the AML phase while assuming Wood Anderson seismogram amplitudes by dividing the amplitude by 2080 (ML_iasp_WA) yields the reported magnitude for the AML phase. Amp is amplitude and $R$ is hypocentral distance.

\begin{tabular}{ccccccc} 
Station & Phase & $\mathbf{R}(\mathbf{k m})$ & $\mathbf{A m p}(\mathbf{n m})$ & Reported $\mathbf{M l}$ & $\mathbf{M l}$ iasp & Ml_iasp_WA \\
\hline \hline LJU & IAML & 504 & 1,504 & 5.4 & 5.0 & 1.7 \\
LJU & AML & 504 & $7,540,000$ & & 8.7 & 5.4 \\
\hline
\end{tabular}

Example 9: Event has wrong IAML amplitudes on BOJS and LJU. Both seem to be 100 times too large.

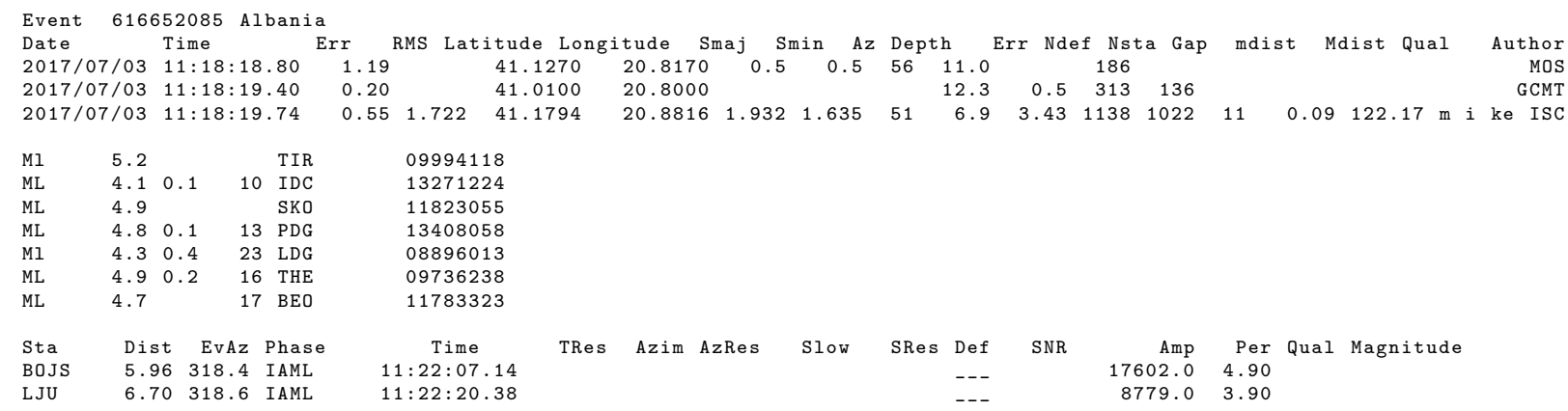

Table 5.3: Calculating $M l$ assuming amplitudes reported as ground displacement (Ml_iasp) gives the wrong magnitude for the two IAML phases while dividing amplitude by 100 (ML_iasp_10̄0) yields the reported magnitudes. Amp is amplitude and $R$ is hypocentral distance.

\begin{tabular}{ccccccc} 
Station & Phase & $\mathbf{R}(\mathbf{k m})$ & $\mathbf{A m p}(\mathbf{n m})$ & Reported Ml & Ml_iasp & Ml_iasp_100 \\
\hline \hline BOJS & IAML & 663 & 17,602 & & 6.5 & 4.5 \\
LJU & IAML & 42 & 8,779 & 6.5 & 4.5 \\
\hline
\end{tabular}

Example 10: Stations TTG (phase Sg) and CEME (Phase Sg) report amplitudes with 4476 and $554 \mathrm{~nm}$ respectively while PUK (phase AMP) and BCI (phase AMP) report 1.0 and $2.9 \mathrm{~nm}$, respectively. TTG and CEME give a reasonable magnitude (average 2.5) using the Hutton and Boore scale (Hutton and Boore, 1987), while PUK and BCO give an average of 0.3. Assuming the amplitudes for PUK and BCO are mm on a Wood Anderson seismogram, we can convert to nm. Now the average magnitude is 2.9 for PUK and BCI. The average Ml reported for this event is 2.4 so 2.9 seems a bit high: Some agencies use the wrong Wood Anderson gain of 2800 instead of 2080, if this is the case, the average magnitude would have been 2.8. In this case the error was easy to spot, but whether the amplitude has been calculated with the correct Wood Anderson gain, or not is still in doubt. This of course is only a problem if the agency reports amplitude in mm on a Wood Anderson seismogram. 


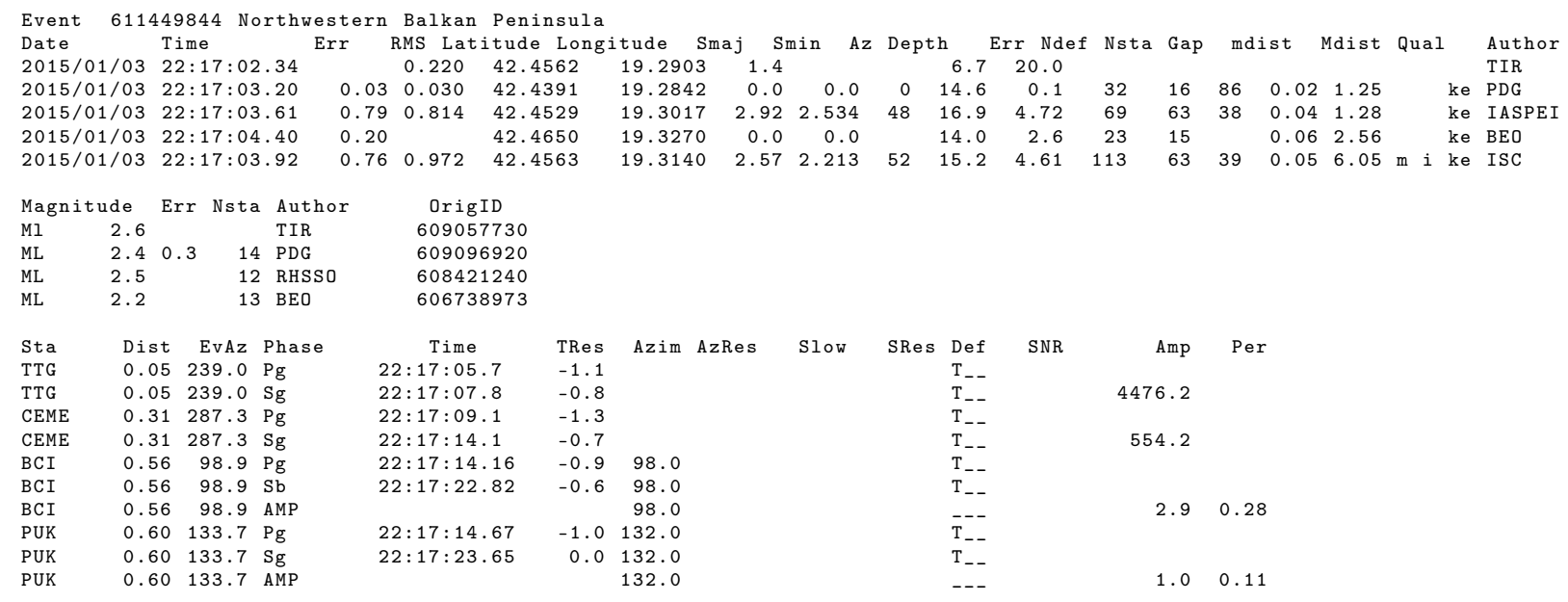

Example 11: All 5 stations that report amplitudes (with $\mathrm{Sg}$ ) give too low $\mathrm{Ml}$ station magnitudes (average value 0.1 ) versus the reported network Ml 1.3. Assuming this to be $\mathrm{mm}$ on a Wood Anderson seismogram, the magnitude would be 2.9 which is too high. So it is not clear what amplitude is reported.

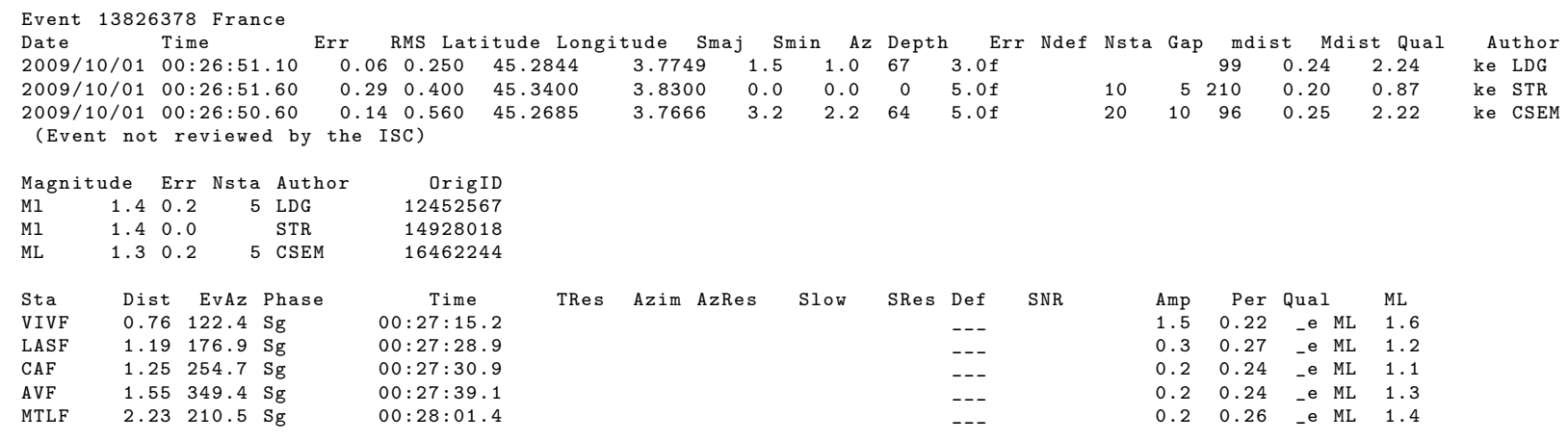

Recommendation: Only use amplitudes from IAML phases. In a few cases even IAML can be wrong though. Use AML phases with caution. If the user wants to use other amplitudes, they should be checked by calculating magnitudes for distances around 50-200 km using the standard California scale and compared to what the corresponding agency has reported.

\section{$\mathrm{mb}$}

In general amplitudes for mb are correct and are easily selected as the ones used by the ISC. The ISC strictly calculates mb only when the distance is larger than 20 degrees so $\mathrm{P}$ amplitudes at shorter distances are not used. The very wide spread SeisComP system (Hanka et al., 2010; https://geofo n.gfz-potsdam.de/software/seiscomp/) uses a magnitude scale mb extended to shorter distances (J. Saul, personal communication, 2016). The IASPEI standard is to use IAmb while an earlier standard was to use AMB, still used by many. In addition, the ISC accepts amplitudes with the phase names P, $\mathrm{pP}, \mathrm{sP}$ and pmax. The non standard pmax (not in the IASPEI phase list) is still reported by Russia (MOS) and China (BJI) for mb but was more common in the past. Ideally only the latest IASPEI recommendation IAmb should be used but that would severely limit the available data. Arrays have 
Example 14: Agency BJI reports magnitudes $\mathrm{mb}$ and $\mathrm{mB}$. We assume $\mathrm{mB}$ is broadband $\mathrm{mB}$ but the event does not show the corresponding IVmb_BB phases and it seems the amplitude is reported as AMB at station WMQ. The magnitude relation for $\mathrm{mB}$ is

$$
m B=\log \left(V_{\max } / 2 \pi\right)+Q(\Delta, h)-3.0,
$$

where $V_{\max }$ is ground velocity in $\mathrm{nm} / \mathrm{s}$ recorded on a broad band sensor proportional to velocity and $Q$ is a correction function dependent on distance $\Delta$ and depth $h$ (Bormann and Dewey, 2014). In the classic relation, $\left(V_{\max } / 2 \pi\right)$ is equivalent to $(A / T)_{\max }$.

Strictly speaking the amplitude should be in velocity $V=410 \mathrm{~nm} / \mathrm{s}$ and then $m B=\log (V / 2 \pi)+$ correction but that is almost the same as assuming displacement $A=410 \mathrm{~nm}$ and calculating $m B=\log (410 \mathrm{~nm} / 5.8 \mathrm{~s})+$ correction. So in this example it is hard to know what unit is used unless we assume it to be displacement since IVmb_BB has not been used. The event has several Chinese stations with similar reports but they are not used by the ISC since the period is too high. So ISC data probably contains more data for $\mathrm{mB}$, but they are hard to find as, if not using IVmb_BB amplitudes, there is no way of knowing how the amplitude was picked. If the period is above $3 \mathrm{~s}$ it is likely that the amplitude is for $\mathrm{mB}$. China uses the $\mathrm{mB}$ magnitude scale regularly (Bormann et al., 2009), but probably reporting displacement and period instead of velocity.

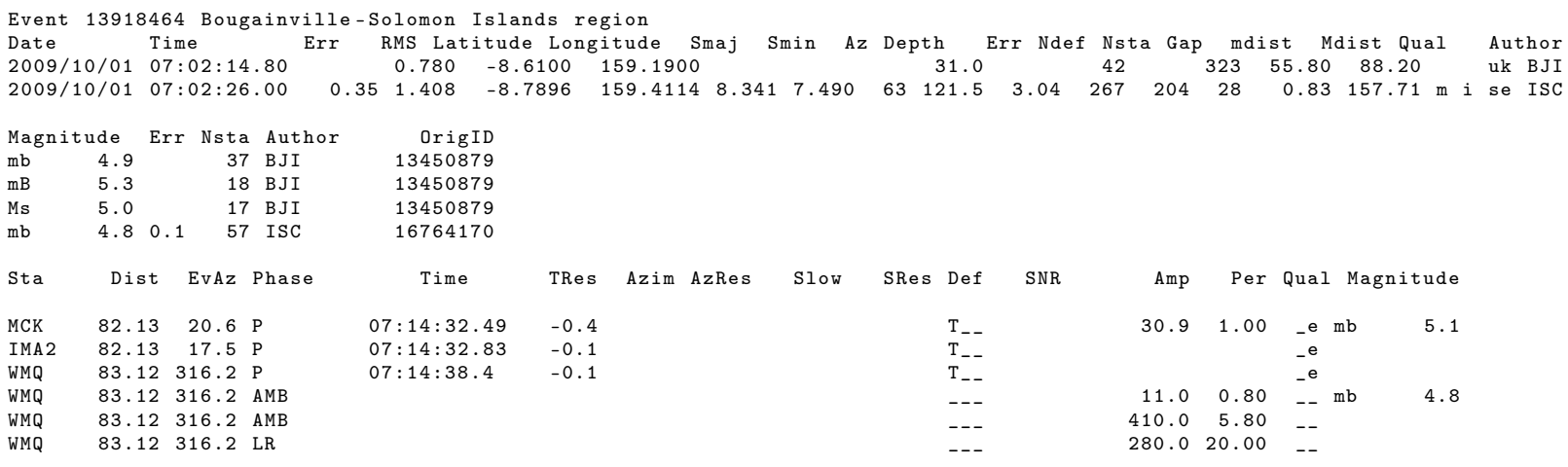

Recommendation: Use all IVmB_BB amplitudes. Amplitudes for $\mathrm{mB}$ with period above $3 \mathrm{~s}$ are most likely in displacement as reporting amplitudes in velocity instead of a displacement and corresponding period suggests that the reporter uses IASPEI standards and thus would likely report the correct phase names.

\section{MS}

Amplitudes for the classic MS are reported with phase names like M, MLR, L, LR and AMS while the IASPEI standard is IAMs_20 indicated that it ideally should be read within the period range 18-22 s. Many amplitudes used by the ISC are not in the 18-22 s range but they are still used as the ISC accepts periods in the range 10 to $60 \mathrm{~s}$.

However, the requirement that the event is shallow (depth $<60 \mathrm{~km}$ ) and in the distance range of 20 to 160 degrees is followed. So MS calculation does not completely follow the IASPEI standard.

Recommendation: Use all amplitudes for which the ISC calculated a MS magnitude and all of the IAMs_20 amplitudes. It is assumed that the user's software automatically does not use data outside 
the valid distance range and depth range. Data with the above phase names can still be included with caution. Abnormal magnitudes must be filtered out by the user's software.

\section{MS BB}

Broadband MS, IASPEI name is Ms_BB, here we use MS_BB: The IASPEI phase name is IVMs_BB indicating that the amplitude is in velocity. Since it can be used in distance range 2 to 160 degrees and period 3 to $60 \mathrm{~s}$ it should have a much wider use. It is particularly useful for larger regional events where $\mathrm{mb}$ and MS cannot be used due to the short distances and Ml being inaccurate. There are very few IVMs_BB reported to the ISC: For November 2018, there are 227, all from SEISAN users. The ISC is not yet using these amplitudes. It seems that BJI calculates MS_BB.

Recommendation: Use all IVMs_BB amplitudes.

\section{Mc or Md}

Coda magnitudes from contributing agencies are given but the coda length is not stored. The IASPEI standard is to mark the end of the coda with phase name END from which the coda length and coda magnitude can be calculated. Only if the END phase is used will the ISC store the coda. However they seem not to be reported very often and the ISC has only about 100,000 observations in total, nearly all from Italy (ROM). The END phase has been implemented in SEISAN version 12.0 so there will probably be more in the future although coda magnitudes are used much less than they used to.

Recommendation: Use all END phases.

\subsubsection{Summary}

Using ISC data presents only a few problems for telseismic phases and magnitudes mb and MS. For local events, where data is often more inhomogeneous, phase names $\mathrm{Pg}, \mathrm{Pn}$ and $\mathrm{Pb}$ should be changed to $\mathrm{P}$ and $\mathrm{S}$ respectively.

Amplitude data for $\mathrm{Ml}$ should be checked before use although most data for IAML phases are OK.

For all phases it is best to use data that is time-defining or has a time residual if relocated by the ISC. Other phases from ISC located events should initially be weighted out. For events not located by the ISC all phases should be used.

\subsubsection{Suggestions for Improved ISC Reporting}

Jens: Calculate residuals for back azimuth, even if not used, so the user can find reliable observations. Or better, start using back azimuth, then maybe more IDC only events could be located by ISC.

Kathrin/ISC: The ISC only recently finished the Rebuild project where we recalculated our entire Bulletin between 1964 to 2010 with ISCloc and ak135 to make the Bulletin consistent (ISCloc was implemented in 2009). Changing our location procedure now and introducing another inconsistency is very 
unlikely to happen for some time. This would be a very non-trivial change that would require a lot of staff time.

Jens: Calculate $\mathrm{Ml}$ for reported amplitudes up to $100 \mathrm{~km}$ and flag values outside reasonable limits. Up to $100 \mathrm{~km}$ there is little difference between different regional scales so the Ml should be reasonable correct. Or better, calculate $\mathrm{Ml}$ using Hutton and Boore and report. Indicate large outliers.

Kathrin/ISC: As described above, $M$ lamplitudes come in a variety of standards that would require a lot of staff time to sort out and confirm. Unfortunately, it is not viable for us to do so at the moment. Should we decide to calculate a Ml_ISC magnitude this might change. In addition, currently ISF has no way to flag magnitude outliers so this would require some format changes.

Jens: Calculate magnitude for broadband MS and mb.

Kathrin/ISC: When time and funding allows we plan to tackle calculating additional magnitudes other than $m b$ and $M S$.

Jens: Make available the phases reported to the ISC also in ISF format so the user can see what has changed.

Kathrin/ISC: As this already is available in QuakeMl format adding it to ISF is not a priority at the moment.

Jens: Flag events not processed by the ISC.

Kathrin/ISC: We are in the process of implementing this.

The ISC is grateful for all feedback. Please contact us for questions, comments and suggestions for improving our data sets and services, or should you find any faults in our data (http://www.isc.ac. $\mathrm{uk} /$ contact/).

\section{Acknowledgments}

We thank James Harris and Domenico Di Giacomo for their help with queries about the ISC database, ISCloc and ISC magnitudes and Natalia Poiata for her helpful comments on the manuscript.

\section{References}

Aki, K. and P. G. Richards (2002), Quantitative seismology, Second Edition, University Science Books, Sausalito, ISBN 0-935702-96-2, 704 pp.

Bondar, I. and D. Storchak (2011), Improved location procedures at the International Seismological Centre, Geophys. J. Int., 186(3), 1220-1244, https://doi.org/10.1111/j.1365-246X.2011.05107. $\mathrm{X}$. 
Bormann, P., R. Liu, Z. Xu, K. Ren, L. Zhang and S. Wendt (2009), First Application of the New IASPEI Teleseismic Magnitude Standards to Data of the China National Seismographic Network, Bull. Seismol. Soc. Am., 99, 1868-1891, https://doi.org/10.1785/0120080010.

Bormann, P. and J.W. Dewey (2014), The new IASPEI standards for determining magnitudes from digital data and their relation to classical magnitudes, In: Bormann, P. (Ed.), New Manual of Seismological Observatory Practice 2 (NMSOP-2), Potsdam, Deutsches GeoForschungsZentrum GFZ, 1-44, https://doi.org/10.2312/GFZ.NMSOP-2_IS_3.3.

Hanka, W., J. Saul, B. Weber, J. Becker, P. Harjadi, Fauzi and GITEWS Seismology Group (2010), Real-time earthquake monitoring for tsunami warning in the Indian Ocean and beyond, Nat. Hazards Earth Syst. Sci., 10, 2611--2622, https://doi.org/10.5194/nhess-10-2611-2010, 2010.

Havskov, J., P. Voss and L. Ottemöller (2020), Seismological observatory software: Thirty years of SEISAN, Seismol. Res. Lett., 91, 1846-1852, https://doi.org/10.1785/0220190313.

Hutton, L. K. and D. M. Boore (1987), The ML scale in southern California, Bull. Seismol. Soc. Am., 77, 2074--2094.

IASPEI, Working Group on Magnitudes, http://www.iaspei.org/commissions/commission-on-sei smological-observation-and-interpretation/Summary_WG_recommendations_20130327.pdf

Kennett, B.L.N, E.R. Engdahl and R. Buland (1995), Constraints on seismic velocities in the Earth from traveltimes, Geophys. J. Int., 122(1), 108-124, https://doi.org/10.1111/j.1365-246X.1995. tb03540.x.

Klein, F. W. (2002), User's guide to HYPOINVERSE-2000, a Fortran program to solve for earthquake locations and magnitudes, Open-File Report 2002-171, US Geological Survey, https://doi.org/10. 3133/ofr02171.

Lienert, B. and J. Havskov (1995), A Computer Program for Locating Earthquakes Both Locally and Globally, Seismol. Res. Lett., 66(5), 26-36, https://doi.org/10.1785/gssrl.66.5.26.

Schweitzer J., D.A. Storchak and P. Borman (2019), Seismic Phase Nomenclature: The IASPEI Standard, In: Gupta H. (eds), Encyclopedia of Solid Earth Geophysics, Encyclopedia of Earth Sciences Series, Springer, Cham, https://doi.org/10.1007/978-3-030-10475-7_11-1.

Stein, S. and M. Wysession (2003), Introduction to Seismology, Earthquakes and Earth Structure, Blackwell Publishing, Oxford, 498 pp.

Storchak, D.A., J. Schweitzer and P. Bormann (2003), The IASPEI Standard Seismic Phase List, Seismol. Res. Lett., 74(6), 761-772, https://doi.org/10.1785/gssrl.74.6.761.

Storchak D.A., J. Schweitzer and P. Bormann (2011), Seismic Phase Names: IASPEI Standard, In: Gupta H.K. (eds) Encyclopedia of Solid Earth Geophysics. Encyclopedia of Earth Sciences Series, Springer, Dordrecht, https://doi .org/10.1007/978-90-481-8702-7_11. 